\title{
A INICIAÇÃO CIENTÍFICA NA VIDA ACADÊMICA: RELATO DE EXPERIÊNCIA
}

\author{
Ana Cláudia de Queiroz ${ }^{1}$, Maria Clara Soares Dantas ${ }^{1}$, Luciana Dantas Farias \\ de Andrade $^{2}$ \\ ${ }^{1}$ Curso de Bacharelado em Enfermagem, Unidade Acadêmica de Saúde, Universidade Federal \\ de Campina Grande, Cuité-PB, Brasil. \\ ${ }^{2}$ Prof ${ }^{a}$ Unidade Acadêmica de Saúde, Universidade Federal de Campina Grande, Cuité, PB, \\ Brasil. \\ Email para correspondência: luciana.dantas.farias@gmail.com
}

\begin{abstract}
Resumo
A Iniciação Científica é a primeira oportunidade do estudante da graduação com a pesquisa acadêmica. Neste sentido, objetivou-se relatar a experiência vivenciada na iniciação científica por duas acadêmicas de um curso de enfermagem de um campus em expansão de uma Instituição Federal de Ensino Superior. Metodologicamente ancora-se no relato de experiência, descrevendo a importância dos conhecimentos, particularidades e experiências vivenciadas na oportunidade de realização de projeto de iniciação cientifica, no período de agosto de 2018 a julho de 2019, por estudantes de enfermagem de uma universidade de Campina Grande, campus Cuité/Paraíba/Brasil. Tal experiência transformadora pessoal e acadêmica a que foi imposta é fruto de inquietações do ser pesquisador, possibilitando uma nova visão de mundo, de subjetividades, de compreensão, de conhecimento, enfrentamento e superação às adversidades, conquistas e toda carga de aprendizado. A inserção dos estudantes em projetos de pesquisa colabora para o processo de educação, como também para o fortalecimento das relações interpessoais e atualização constantes, no qual revelou a importância da iniciação científica para o desempenho acadêmico em relação à dedicação, responsabilidade, criatividade, persistência e conhecimentos adquiridos, além da valorização da motivação, determinação e resiliência.
\end{abstract}

Palavras-chave: Enfermagem; educação; ciência.

\begin{abstract}
/ resumen / résumé
Scientific Initiation is the first opportunity of the undergraduate student with academic research. In this sense, the objective was to report the experience lived in scientific initiation by two students of a nursing course in an expanding campus of a Federal Institution of Higher Education. Methodologically it is anchored in the experience report, describing the importance of the knowledge, particularities and experiences lived in the opportunity to carry out a scientific initiation project, from August 2018 to July 2019, by nursing students from a university in Campina Grande , campus Cuité / Paraíba / Brazil. Such a transformative personal and
\end{abstract}


academic experience to which it was imposed is the result of the researcher's concerns, enabling a new worldview, subjectivities, understanding, knowledge, coping and overcoming adversities, achievements and all learning load. The inclusion of students in research projects contributes to the education process, as well as to the strengthening of interpersonal relationships and constant updating, in which it revealed the importance of scientific initiation for academic performance in relation to dedication, responsibility, creativity, persistence and acquired knowledge, in addition to valuing motivation, determination and resilience.

Keywords: Nursing; education; science.

\section{Introdução}

A Iniciação Cientifica (IC) é a primeira oportunidade do estudante da graduação com a pesquisa acadêmica, podendo continuar nos programas de pósgraduação. Pode ser considerada imprescindível na política de pesquisa das várias Instituições de Ensino Superior (IES) e pode ser disponibilizada em duas modalidades, a modalidade "bolsa", que é a oferta de um valor monetário pago ao aluno, como forma de incentivo financeiro, ou na modalidade "voluntária", cujo auxílio é predominantemente pedagógico, geralmente tem duração de um ano e pode ser a oportunidade para viabilizar, o mais precocemente possível, o contato com a importante demanda científica e envolvimento na pesquisa (PIRES, 2015).

Fazer ciência é um processo de construção da autonomia, é tornar-se sujeito de seu processo de aprendizagem. Universidades e Centros de Pesquisa, na condição de formadores de recursos humanos, compartilham a tarefa de difundir cultura de responsabilidade, tanto ética como científica. As Instituições de Ensino são espaços de formação e transformação, que, além de qualificar profissionais e cientistas no âmbito teórico, assumem a importante função de proporcionar experiências práticas de construção do saber, de reflexão e de desenvolvimento de independência (SILVA et. al., 2020).

Os estudantes da graduação podem considerar a carreira laboral de pesquisador por meio da experiência na IC e, dentre tantos benefícios, destaca-se a produção do conhecimento e a validação do corpo técnico e científico da Instituição (GABRIOTI, 2020).

Estudo demonstrou que a análise realizada em um conjunto de pesquisas, utilizando a metodologia da revisão integrativa, apontou para a existência de um amplo consenso sobre o papel relevante que a IC desempenha na formação universitária, principalmente no que diz respeito às 
atividades acadêmicas realizadas no curso de graduação, ao desenvolvimento pessoal dos alunos, à construção de uma nova visão de ciência e à socialização profissional. Existe, da mesma forma, um consenso sobre os importantes objetivos alcançados pelos programas de fomento à IC, particularmente o Programa Institucional de Bolsas de Iniciação Científica (PIBIC), tendo em vista que despertam e incentivam a vocação científica dos universitários, além de favorecer a ampliação e a consolidação de núcleos e grupos de pesquisa nas Instituições de Ensino Superior (MASSI; QUEIROZ, 2010).

Considerando as limitações financeiras que as IES vem enfrentando que tem ameaçado o número de bolsas ofertadas e considerando as dificuldades vivenciadas pelos pesquisadores para realizar as pesquisas envolvendo 0 respeito aos preceitos éticos, financiamento para as despesas com a realização da pesquisa, bem como para a publicação da mesma, são vários os desafios enfrentados pelos pesquisadores e acadêmicos (NORTE, 2020).

Neste sentido, surgiu o interesse em refletir sobre o tema com a seguinte questão norteadora: Qual a opinião de duas acadêmicas de um curso de enfermagem acerca da experiência vivenciada na iniciação científica?

Entendendo a importância do tema para a formação dos universitários, este estudo tem o objetivo de relatar a experiência vivenciada na iniciação científica por duas acadêmicas de um curso de enfermagem de um campus em expansão de uma Instituição Federal de Ensino Superior.

\section{Materiais e métodos}

Trata-se de um relato de experiência realizado no contexto do desenvolvimento do Programa Institucional de Bolsas de Iniciação Científica (PIBIC) e Programa Institucional de Voluntários de Iniciação Científica (PIVIC), da Universidade Federal de Campina Grande, campus Cuité/Paraíba/Brasil, compreendendo o período de agosto de 2018 a julho de 2019.

Participaram dessa experiência duas acadêmicas do terceiro ano do Curso de Enfermagem, campus Cuité/Paraíba, e a docente que submeteu dois projetos aprovados pela Pró-reitoria de Pesquisa e Extensão (PROPEX) da Universidade Federal de Campina Grande, vigência 2018-2019, que 
vivenciaram as potencialidades e limitações acadêmicas, de modo a permitir a construção de um empreendimento que pudesse expor a visão de mundo dos sujeitos em seu microespaço de ação.

A análise dos dados foi realizada por meio de uma exposição reflexiva, de forma descritiva e associada a estudos com foco na iniciação científica como complemento da formação acadêmica em saúde e enfermagem.

\section{Resultados e discussão}

\subsection{Programa Institucional de Bolsas de Iniciação Científica (PIBIC)}

Após aprovação do projeto na PROPEX, com a benesse do auxílio monetário da bolsa, o primeiro momento é felicidade, mais uma etapa vencida com novo desafio a ser superado. $O$ desenvolvimento de um projeto exige dedicação e motivação.

O cronograma apresentado em duas partes: a primeira contemplava a pesquisa bibliográfica da gestão em saúde e enfermagem por meio de leituras em artigos científicos e livros para se familiarizar com o referencial teórico e a segunda parte convergia para o estudo do referencial metodológico envolvendo o materialismo histórico e dialético, o MHD.

Pode-se apontar a importância em conhecer todas as etapas do desenvolvimento de uma pesquisa científica, pois houve ênfase na construção de um projeto, desde os conceitos basilares, metodologia, coleta de material, organização dos resultados, análise e divulgação.

Semanalmente aconteciam reuniões presenciais conferindo no cronograma das atividades que já haviam sido cumpridas e planejando estratégias para alcançar as demais metas. Houveram imprevistos que prejudicaram a execução do planejamento como atraso para o início da coleta de material empírico e produção do relatório final, mas gradativamente foram sendo sanados por meio do aumento de horas destinadas aos estudos conceituais e metodológicos e realização de coleta de material empírico aos sábados e domingos, sem comprometer bruscamente 0 cronograma inicalmente elaborado.

Um aspecto limitador enfrentado foi o Comitê de Ética em Pesquisa com Seres Humanos (CEP), pois no cronograma foi estimado um prazo para 
aprovação do projeto que, infelizmente, não foi contemplado. Entende-se que há poucos professores para a análise dos projetos no contexto do CEP e, por esta razão, atrasos na emissão do parecer.

Os aspectos conceituais foram trabalhados de maneira satisfatória, uma vez que havia o aprofundamento do arcabouço teórico ministrado pela docente em sala de aula. Isso significa que foi bastante produtivo dedicar tempo para conhecer as publicações mais recentes envolvendo gestão em saúde e enfermagem.

No tocante ao aspecto metodológico, foi estudado com o máximo de dedicação possível os preceitos do Materialismo Histórico e Dialético, MHD, esse método contribue significativamente para a sociedade, pois trabalha a contradição por defender que para pensar a realidade é preciso elucidar os seus extremos, para se aproximar do essencial deve-se partir do cotidiano, do dia-a-dia, dos acontecimentos empíricos para então convergir ao seu aspecto mais abstrato (MINAYO, 2007).

Foi bastante complicado entender essa metodologia porque a leitura do material bibliográfico aborda assuntos que não são normalmente trabalhados na graduação. Debruçar-se em conceitos filosóficos, conhecer pensadores como Karl Marx e Frederic Engels foram desafiadores. Foi preciso muita disciplina, motivação e dedicação para entender um pouco esse universo.

Outra estratégia utilizada, enquanto era aguardado o parecer técnico do CEP, foi ensaiar as entrevistas, revisar o roteiro semiestruturado, estudar como seria o contato com os entrevistados, estudar todo rigor necessário para a coleta de dados e aprofundar as leituras da Resolução ㄲo 466, de 12 de dezembro de 2012 que institui as diretrizes e normas regulamentadoras para realização de pesquisas científicas envolvendo seres humanos (BRASIL, 2012).

Faz-se importante destacar que, apesar da ansiedade em iniciar a coleta de dados e respeitar o cronograma inicialmente elaborado, prioritariamente deve-se atender aos preceitos éticos e em momento algum foi cogitado o início da coleta sem o parecer técnico do CEP.

O Conselho Nacional de Desenvolvimento Científico e Tecnológico (CNPq) foi desenvolvido no Brasil em 1951 como pioneiro no financiamento de 
atividades científicas oferecendo modalidades como o PIBIC, com o objetivo de apoiar a política de iniciação científica desenvolvida nas universidades. Esta iniciativa é capaz de estimular o universitário a aproximar-se de sua realidade social, despertando-o a desenvolver pesquisas ainda na academia, contribuindo tanto para a formação profissional dos estudantes como para a atualização da ciência por meio do estudo constante da sociedade (SANTOS; ANJOS; ALMEIDA, 2015).

O fato da bolsa PIBIC proporcionar a participação constante na produção científica, responsabilidade perante a mesma, oportunidades para desenvolver habilidades, recriar conceitos não entendidos, participação em eventos que divulguem os resultados do estudo, elaboração de artigos científicos, conduz o estudante ao pensamento crítico da realidade (NOVAIS; SILVA, 2011, SOBRAL; SANTOS; TORALES, 2016).

Acrescenta-se que a bolsa de pesquisa possibilita aos acadêmicos passar da condição de passivos para uma condição de sujeitos que se tornam mais ativos, permitindo a sua atuação como investigador de determinada temática, possibilitando, assim, uma importância maior na sua carreira acadêmica, tornando a trajetória do estudante dentro da academia mais importante e relevante (NOVAIS; SILVA, 2011).

Apesar da importância, a própria iniciação científica poderia ser priorizada no Brasil, uma vez que uma pesquisa demostrou que, de maneira geral, todos os estudantes que tiveram essa oportunidade na graduação têm apresentado melhor desempenho nos processos seletivos para a pósgraduação, adquirem mais rápido a titulação stricto sensu, possuem um espírito de equipe, gestão e liderança diferenciados, dispõem de uma maior facilidade ao falar em público e de se adaptar às atividades didáticas. No entanto, Silva (2012) relata que a cada ano, as pesquisas estão sendo cada vez menos incentivadas.

Torna-se evidente que a iniciação científica resulta em uma formação mais qualificada, competente e hábil, já que são exigidas competências que geralmente não são ofertadas em sala de aula, além disso, para a participação dos estudantes nos programas institucionais é preciso um rendimento escolar satisfatório, exigindo ainda mais dedicação e disciplina (NARDINI et al., 2019). 
Com o objetivo de conhecer os obstáculos existentes no processo de produção científica em saúde, pesquisa ancorada no método qualitativo por meio de entrevistas realizadas com os profissionais de saúde de uma coordenadoria da cidade de Fortaleza, Ceará, e em um serviço da atenção primária, no período de 2015 e 2016, revelou limitações em relação à Universidade e aos serviços de saúde tais como: questões burocráticas para entrada em campo, atraso para início da coleta de dados e as dificuldades do serviço em acolher os pesquisadores. Apesar das limitações à realização do estudo não impediram que fosse finalizado, embora tenha desmotivado professores e alunos (PAULA; JORGE; MORAIS, 2019).

O relatório Research in Brazil, material elaborado pela Clarivate Analytics para a Coordenação de Aperfeiçoamento de Pessoal de Nível Superior (CAPES), descreve uma análise sucinta do desempenho da pesquisa brasileira entre os anos de 2011 a 2016 e revela que o Brasil aparece em 13ำ lugar no ranking das publicações mundiais, à frente de todos os outros países da América Latina. No entanto, o impacto da citação dos documentos brasileiros é baixo em relação à média mundial, está centralizada em alguns Estados, principalmente São Paulo, e os pesquisadores brasileiros têm trabalhado mais em parcerias internacionais, gerando um impacto maior que a pesquisa nacional, para poder alcançar níveis elevados (MOURA, 2018).

Embora o Brasil tenha melhorado o desempenho nos últimos anos, o país ainda tem menos cientistas em relação à população total do que a maioria dos países desenvolvidos, há baixa qualidade da educação fundamental, as multinacionais que atuam no Brasil resistem em colaborar com grupos de pesquisa brasileiros e a produção brasileira precisa melhorar no tocante à internacionalização, com perspectivas sombrias para o futuro (ZIEGLER, 2018).

\subsection{Programa Institucional de Voluntários de Iniciação Científica (PIVIC)}

Após realização do processo seletivo para aprovação do candidato ao projeto de iniciação científica sem remuneração monetária, houve reunião entre docente e discente para organização do cronograma a ser executado. 
Infelizmente, após um mês de trabalho, este estudante precisou trancar o curso por motivos pessoais convergindo à convocação do segundo colocado.

Após a devida regulamentação dos trâmites burocráticos para atualização no PROBEX, foi possível iniciar as reuniões semanais, atualização do cronograma previamente elaborado e continuar a revisão integrativa que havia sido iniciada.

A primeira parte do cronograma consistia no estudo conceitual pela necessidade de estimular a leitura e análise dos diversos estudos que perpassam a gestão em enfermagem. Após estudo conceitual seguiu-se ao estudo metodológico, com leituras acerca da construção de uma revisão integrativa da literatura.

Foi uma experiência enriquecedora, por permitir aprender a importância da paciência, do lento processo que envolve a aprendizagem, infinitude do conhecimento científico, resiliência, enfrentamento das adversidades e valorização das pequenas conquistas.

Em relação ao desenvolvimento pessoal, pesquisa aponta qualidades que são adquiridas com a experiência promovida pelo desenvolvimento da pesquisa para futura vida profissional, seja na prestação de serviços à sociedade ou Universidade, dentre as quais destacam-se o raciocínio crítico, autonomia, criatividade, maturidade e responsabilidade. (MASSI; QUEIROZ, 2010).

O conhecimento acerca da inserção precoce do graduando na IC é um instrumento valioso para aprimorar atributos desejados em um profissional de nível superior, como também despertar a vocação para a pesquisa, possibilitanto um direcionamento mais antecipado dos estudantes para a pósgraduação stricto sensu (NARDINI et al., 2019).

Esse caminho de estreitamento das relações entre graduação e pósgraduação que a IC proporciona como ferramenta educacional permite não apenas o empoderamento do estudante em suas escolhas profissionais, como desperta habilidades e competências que poderão ser melhor aproveitadas (FERREIRA NETO, 2017).

Pesquisa desenvolvida no Departamento de Enfermagem da Universidade Estadual de Montes Claros no mês de maio de 2017 objetivou 
analisar o perfil dos enfermeiros-docentes da Graduação por meio de um estudo descritivo, do tipo survey, com dados extraídos de 57 currículos. A maioria dos profissionais apresentaram graduação em instituições formadoras públicas, mais de 12 anos de experiência na docência e mais de 15 anos de atuação laboral em Estabelecimentos Assistenciais de Saúde públicos, filantrópicos e privados, titulação de mestres e doutores, publicações em periódicos qualis com menor fator de impacto. Os autores concluíram que a experiência e titulação qualificam os docentes para a assistência e/ou docência refletindo a preocupação com a qualidade do cuidado a ser ofertado ao paciente, no entanto, recomendaram a inserção destes profissionais em grupos de pesquisa com estímulo à iniciação científica como importantes estratégias para qualificação, reconhecimento e melhora das publicações em enfermagem (ALMEIDA et al., 2020).

\section{Conclusão}

Vários desafios são enfrentados para realização de pesquisas no Brasil, dentre eles: limitações financeiras que as universidades vem enfrentando, limitações financeiras dos professores, diminuição no número de bolsas, dificuldades para realização da pesquisa em termos de autorização e início da coleta de dados, acesso a computadores, programas e softwares, despesas com a publicação, exigência de visibilidade internacional, dentre outras.

Mesmo diante de tantos desafios, as pesquisas continuam sendo realizadas, professores continuam otimistas em relação à confecção científica e cenários promissores para a realidade que vem sendo enfrentada. Não foi diferente em relação à realização deste estudo.

A experiência vivenciada por duas acadêmicas de enfermagem revelou a importância da iniciação científica para o desempenho acadêmico em relação à dedicação, responsabilidade, criatividade, persistência e conhecimentos adquiridos, além da valorização da motivação, determinação e resiliência.

Por esta razão, estudos que objetivem pesquisar os desafios para realização da iniciação científica precisam ser realizados para traçar estratégias de enfrentamento às adversidades, apoio aos jovens pesquisadores e incentivo às pesquisas em território nacional. 


\section{Referências}

ALMEIDA, E. W. S. et al. Perfil dos enfermeiros-docentes em uma universidade pública: em que precisamos avançar? Rev. Pesqui. (Univ. Fed. Estado Rio J., Online), v. 12, p. 559-565, jan.-dez., 2020.

BERALDO, G. S.; FERREIRA NETO, J. L. Iniciação científica na formação em psicologia: uma revisão de literatura. Psicologia em revista, Belo Horizonte, v. 23, n. 3, p. 1034-1050, dez., 2017. Disponível em: http://pepsic.bvsalud.org/pdf/per/v23n3/v23n3a15.pdf. Acesso em: 05 jun. 2020.

BRASIL. Ministério da Saúde. Conselho Nacional de Saúde. Resolução № 466 de 12 de dezembro de 2012. Dispõe sobre pesquisas e testes em seres humanos. 2012. Disponível em: http://conselho.saude.gov.br/ultimas_noticias/2013/06_jun_14_publicada_resolucao.ht ml Acesso em: 28 mar 2018.

GABRIOTI, R. Bolsas de estudo no Brasil: uma modalidade de fomento FAPESP à pesquisa em comunicação. Intercom - RBCC, São Paulo, v. 43, n. 1, p.109-128, jan./abr., 2020. Disponível em: <https://www.scielo.br/pdf/interc/v43n1/1809-5844interc-43-1-0109.pdf>. Acesso em: 17 mar. 2020.

MASSI, L.; QUEIROZ, S. L.. Estudos sobre iniciação científica no Brasil: uma revisão. Cadernos de Pesquisa, v. 40, n. 139, p.173-197, jan./abr., 2010. Disponível em: <https://www.scielo.br/pdf/cp/v40n139/v40n139a09.pdf>. Acesso em: 21 jul. 2020.

MINAYO, M. C. S. O Desafio do conhecimento: pesquisa qualitativa em saúde. 10. ed. São Paulo: Hucitec/Abrasco, 2007.

MOURA, E. Pesquisa científica no Brasil: o que deu errado? 2018. Disponível em: <https://pebmed.com.br/pesquisa-cientifica-no-brasil-o-que-deu-errado/>. Acesso em: 17 jul. 2020.

NARDINI, E. F. et al. Política de estímulo à iniciação científica: impacto no coeficiente de rendimento de graduandos em Odontologia. Rev. Abeno, v. 19, n. 1, p. 33-39, 2019. Disponível em:

<https://revabeno.emnuvens.com.br/revabeno/article/view/619/524>. Acesso em: 05 jun. 2020.

NORTE, D. B. Cortes e mais cortes: o que será da ciência e da pesquisa no Brasil? 2020. Disponível em: <https://vocesa.abril.com.br/carreira/cortes-bolsas-pesquisa-ciencia/> Acesso em: 23 mai. 2020.

NOVAIS , N. N.; SILVA, L. W. S. O ambiente da investigação em Enfermagem: um relato de experiência. Rev. Bras. Enferm., Brasília (DF), v. 64, n. 3, p. 605-610, mai./jun., 2011. Disponível em <http://www.scielo.br/pdf/reben/v64n3/v64n3a29.pdf>. Acesso em: 21 mar. 2020.

PAULA, M. L. de; JORGE, M. S. B. MORAIS, J. B. de. O processo de produção científica e as dificuldades para utilização de resultados de pesquisas pelos profissionais de saúde. Interface. Botucatu, v. 23, ago., 2019. Disponível em: 
$<$ https://www.scielo.br/scielo.php?pid=S1414-

32832019000100271\&script=sci_arttext\&tlng=pt>. Acesso em: 16 jul. 2020.

PIRES, R. C. M. O trabalho do professor-pesquisador e o PIBIC/CNPQ. In: MASSI, Luciana; QUEIROZ, Salete Linhares. Iniciação científica: aspectos históricos, organizacionais e formativos da atividade no ensino superior brasileiro [online]. São Paulo: Editora UNESP, 2015, p. 87-108.

SANTOS, V. C.; ANJOS, K. F. dos; ALMEIDA, O. S. Iniciação Científica a partir de Estudantes de Enfermagem. Rev. Bras. Ciênc. Saúde, v. 19, n. 4, p. 255-260, 2015. DOI:10.4034/RBCS.2015.19.04.01. Disponível em:

$<$ https://periodicos.ufpb.br/ojs/index.php/rbcs/article/view/19991/15099>. Acesso em: 05 Jun de 2020.

SILVA, L. F. F. Iniciação científica - contexto e aspectos práticos. Rev. Med. São Paulo, v. 91, n. 2, p. 128-136, abr./jun., 2012. Disponível em:<http://www.revistas.usp.br/revistadc/article/view/58973/61960>. Data de Acesso: 05 Jun de 2020.

SILVA, N. R. A. et al. Integridade científica entre alunos de enfermagem que participam do Programa de Iniciação Científica: Estudo exploratório. Rev Esc Enferm USP, v. 54, e03548, 2020. Disponível em:

$<$ https://www.scielo.br/pdf/reeusp/v54/pt_1980-220X-reeusp-54-e03548.pdf >.Data de Acesso 17 Mar de 2020.

SOBRAL, H. C. F; SANTOS, I. H. F.; TORALES, A. P. B. Relato de experiência: A Iniciação Científica Na Vida Acadêmica Experiência Acadêmica. 8 Encontro internacional de formação de professores, Sobral, v. 9, n. 1, 2016. Disponível em: $<$ https://eventos.set.edu.br/index.php/enfope/article/viewFile/2450/539>. Acesso em: 21 mar. 2020.

ZIEGLER, M. F. Livro aborda dificuldades para inovação no Brasil. 2018.

Disponível em: <http://agencia.fapesp.br/livro-aborda-dificuldades-para-a-inovacao-nobrasil/28065/>. Acesso em: 10 jul. 2020.

\section{Agradecimentos}

Agradecimentos especiais ao Conselho Nacional de Desenvolvimento Científico e Tecnológico (CNPq), Coordenação de Aperfeiçoamento de Pessoal de Nível Superior (CAPES) e Universidade Federal de Campina Grande (UFCG) pela construção de novos saberes e por viabilizar a oportunidade de buscar a excelência na formação científica. 